\title{
¿Podría ser aceptable la eutanasia infantil?
}

\author{
J.P. Beca • A. Leiva
}

\section{ABSTRACT \\ Could infant euthanasia be ever acceptable?}

The recent enactment of a law that allows infant euthanasia in Belgium raises questions with varied answers. To contribute to a better understanding of the topic, euthanasia and legislation concepts are described. After a bioethical analysis, we propose as conclusion that children euthanasia could only be acceptable in very exceptional situations in which palliative measures have failed. The answer should be that it is not acceptable in our setting, not until we have public policies, protocols and palliative care services for terminally ill children.(Key words: Euthanasia, palliative care, suicide, terminal, illness).

Rev Chil Pediatr 2014; 85 (5): 608-612

\section{RESUMEN}

La reciente promulgación de una ley que permite la eutanasia infantil en Bélgica plantea interrogantes que admiten respuestas diversas. Para contribuir a una mejor comprensión del tema se describen los conceptos de eutanasia y la legislación pertinente. Después de hacer un análisis bioético, se plantea como conclusión que la eutanasia de niños podría ser aceptable sólo de manera muy excepcional ante situaciones en las cuales hubiesen fracasado las medidas de cuidado paliativo. Para nuestro medio la respuesta debería ser que no es aceptable, al menos mientras no existan políticas públicas, protocolos y servicios de cuidados paliativos para niños con enfermedades terminales.

(Palabras clave: Eutanasia, cuidados paliativos, suicidio, enfermedad terminal).

Rev Chil Pediatr 2014; 85 (5): 608-612

Si la eutanasia plantea serias dudas y permanente discusión sobre su posible aceptación, tanto moral como legal, cuando se trata de niños el problema es aún mayor. Recientemente y en febrero de este año, el parlamento belga ha aprobado una ley que permite la apli- cación de la eutanasia en niños, sin distinción etaria. La norma en comento modifica la ley anterior (2002) que permite la eutanasia para adultos, extendiéndola a los menores ${ }^{1}$. Bélgica pasa así a ser el segundo país del mundo, luego de Holanda (2001) en permitir la eutanasia en

Recibido el 17 de abril de 2014, última versión aceptada el 25 de agosto de 2014.

Juan Pablo Beca I. ( $ه)$

Centro de Bioética, Facultad de Medicina, Clínica Alemana-Universidad del Desarrollo.

E-mail:jpbeca@mi.cl

Alejandro Leiva L.

Facultad de Derecho, Universidad del Desarrollo. 
niños. Estas legislaciones plantean problemas en su justificación y actitudes de apoyo o de rechazo, generalmente con escasa comprensión de las razones en las que ellas se han basado.

Para contribuir a una mejor comprensión del tema, en este artículo se revisan el concepto de eutanasia, las leyes de despenalización existentes, en especial el caso de Holanda y Bélgica, los problemas bioéticos y jurídicos en su regulación, y las razones para aceptarla o para rechazarla.

\section{Concepto de Eutanasia y la nueva ley de eutanasia infantil en Bélgica}

Como concepto de eutanasia, más allá de su significado etimológico de "buena muerte", se han asumido diferentes definiciones y calificaciones o adjetivos que dificultan la discusión del tema. Para mayor claridad, en este análisis se asume como definición clínica la siguiente: "en el contexto clínico, consiste en todas aquellas acciones realizadas deliberadamente por un médico, u otro profesional sanitario bajo su dirección, que tienen como objetivo provocar directamente la muerte a petición expresa, voluntaria y reiterada de un paciente capaz que presenta un sufrimiento intenso, físico o psíquico, a consecuencia de una enfermedad terminal o irreversible y que el propio paciente experimenta como algo inaceptable, insoportable e indigno" ${ }^{2,3}$. Si bien, en el ámbito jurídico, algunos consideran también como eutanasia las omisiones y tratamientos con la intención de producir la muerte ${ }^{4}$, en este comentario se dejará de lado esta discusión.

En materia normativa, debemos señalar que la eutanasia no está tipificada expresamente en la ley chilena, lo cual no excluye la responsabilidad criminal en que puede incurrir el facultativo que la lleve a cabo. Ello se explica, como se ha señalado, ya que la eutanasia importa provocar la muerte de una persona, en este caso la de un paciente, conducta que se enmarca dentro del tipo de homicidio que se define como "el que mate a otro", independiente del estado de salud del paciente o de su voluntad expresada para autorizar su muerte. Por lo tanto si un profesional sanitario es quien provoca la muerte de un paciente estaremos frente a dicho ilícito. Asimismo, y tratándose de la eutanasia aplicada a un recién nacido con menos de $48 \mathrm{~h}$ de vida, el tipo aplicable será el de infanticidio, consagrado en el artículo 394 del Código Penal. En definitiva, no existe un concepto legal o penal de la eutanasia, pues ella no ha sido reconocida expresamente en nuestra legislación. Sin perjuicio de ello, en materia de dogmática penal, se ha definido la eutanasia como: "la ayuda prestada a una persona gravemente enferma, por su deseo o por lo menos en atención a su voluntad presunta, para posibilitarle una muerte humanamente digna en correspondencia con sus propias convicciones"5.

En cuanto a las legislaciones de Holanda y Bélgica, las condiciones para que se autorice legalmente a efectuar la eutanasia en adultos son las siguientes: enfermedad terminal, sufrimiento que no se logra relevar con los tratamientos existentes, petición voluntaria y reiterada del paciente, y al menos dos opiniones médicas que confirman la situación clínica y la capacidad de decisión del paciente.

La nueva normativa belga que autoriza la eutanasia infantil, consagra que el menor, independientemente de su edad, puede optar por poner término a su vida bajo determinadas circunstancias. Así, deberá: 1) ser sometido a un examen psicológico que confirme su capacidad de discernimiento para tomar tal decisión; 2) acreditarse que se encuentra bajo un constante e insoportable dolor que no puede ser aliviado, 3) que éste resulta de una condición de sufrimiento físico accidental o patológico grave e incurable, siendo su muerte a corto plazo inevitable, y 4) serán los padres quienes deberán autorizar la eutanasia en forma expresa y por escrito antes de que ésta pueda ser aplicada. De esta forma, y cumpliéndose dichos requisitos, podrá procederse a la eutanasia de cualquier menor.

La ley holandesa, en cambio, no autoriza en ningún caso la eutanasia en menores de 12 años. Pero sí la autoriza en mayores de 12 y menores de 16 años, siempre y cuando los padres presten su autorización en forma expresa, y en mayores de 16 y menores de 18 años, simplemente oyendo a los padres. 


\section{Análisis ético del tema}

Antes de analizar las razones y argumentos en favor o en contra, parece necesario dejar en claro cuál es el problema de fondo por el cual se plantea la eutanasia infantil. No se trata, como en otras épocas, de situaciones de eugenesia o discriminación, sino de niños con enfermedad terminal con grave sufrimiento, y sin que las medidas paliativas logren suprimirlo, en situaciones en las cuales los mismos niños piden no seguir viviendo, bajo la condición de que sus padres estén de acuerdo. Se trata pues de un grave problema clínico y humano para el cual no tenemos respuestas unívocas, situación en la que surge la eutanasia como una alternativa posible.

Para algunos, la eutanasia resulta inaceptable en cualquier circunstancia y a cualquier edad, sobre la base de que nunca se puede atentar contra una vida humana por su valor intrínseco absoluto o por su sacralidad. Quienes así piensan, ya tienen una posición definitiva ante este nuevo escenario de eutanasia en niños. Sin embargo, no deberían asumir que quienes la aceptan lo hacen sin reconocer el valor de la vida de los niños en fase terminal, ni deberían descalificar a quienes piensan de manera diferente. Por el contrario, quienes están abiertos a aceptar la validez de la eutanasia en casos excepcionales en adultos, necesitan reflexionar ahora sobre este nuevo escenario de la eutanasia infantil, para fundamentar su rechazo o su aceptación.

Las decisiones al final de la vida en niños con enfermedad terminal son particularmente complejas y difíciles para los profesionales involucrados y para los padres de estos pacientes. Un adecuado razonamiento hace necesario considerar los significados de la vida, de la enfermedad, del sufrimiento y de la muerte, temas que rara vez se enfrentan. La consecuencia es que, por temor a facilitar la muerte, las decisiones se postergan aplicando medidas que prolongan artificialmente el proceso de morir, aumentando así el sufrimiento ${ }^{6,7}$. Es lo que conocemos como obstinación terapéutica ${ }^{8}$ y que desde un enfoque bioético principialista atenta contra el principio de No Maleficencia.

Las alternativas al final de la vida no se pueden reducir a aceptar o no la eutanasia, sino a plantearse un conjunto de medidas destinadas a aliviar el sufrimiento con un enfoque holístico y no limitado al dolor físico. En este sentido, lo primero será adecuar o limitar tratamientos que no logran un objetivo terapéutico y que son, por lo tanto, fútiles o desproporcionados, con lo cual claramente se acepta o permite la muerte sin buscar producirla ${ }^{9}$. Este tipo de decisiones se diferencian sustancialmente de formas de abandono del paciente, en la medida en que se acompañan del mejor cuidado básico y control de síntomas, como el dolor y la angustia. Se complementa este enfoque con medidas de cuidado emocional y espiritual del niño enfermo según su edad, de sus hermanos y de sus padres. Resulta lógico pensar que si el final de la vida de los niños se asume profesional y familiarmente de esta manera, el espacio para casos en que se plantee la eutanasia será mínimo. Pero siempre habrá casos excepcionales en los cuales, a pesar de todos los recursos disponibles, el sufrimiento no es controlable, la enfermedad es progresiva o presenta agudizaciones graves y la calidad de vida es realmente mala.

Las razones para a aceptar la eutanasia en adultos se fundamentan principalmente en el respeto a la autonomía del paciente, de acuerdo con la cual cada enfermo es dueño de su vida y de definir cuándo la prolongación de la vida resulta le resulta peor que la muerte ${ }^{10}$. Para algunos, si se le reconoce al enfermo este derecho, resulta coherente reconocerle el derecho no sólo a rechazar tratamientos que únicamente prolongan su vida, sino también el derecho a pedir que se ponga fin a su vida. Para otros, el aceptar éticamente la eutanasia, resulta contradictorio porque reconocer el principio de autonomía para disponer de la propia vida sería negar el mismo principio ${ }^{11}$.

Pero como esta es una decisión muy seria y definitiva, para aceptarla es necesario asegurarse previamente que ya no es posible el control de síntomas, particularmente del dolor y de la angustia, que el enfermo no esté patológicamente deprimido y que su petición sea reiterada y no meramente ocasional. Si bien lo anterior puede resultar razonable para las solicitudes de eutanasia en casos excep- 
cionales y debidamente controlados en adultos, en el caso de niños se plantean nuevos problemas.

Para considerar o no éticamente aceptable la eutanasia infantil se requiere asumir antes una visión sobre el valor de la vida del niño, aun en condiciones de enfermedad y sufrimiento. Se trata de una pregunta metafísica sobre el significado de la vida, ante la cual no hay respuestas universales y por tanto, quienes deciden ante casos particulares no pueden imponer sus propias creencias.

Un segundo aspecto a considerar es la capacidad de decisión del niño, lo cual significa respetar su autonomía en la medida en que sea capaz de decidir. Esta evaluación de capacidad, exigida en la ley belga, es de particular dificultad dada la variabilidad de madurez de los menores y con mayor razón de los menores que tienen ya la experiencia de la enfermedad y de la cercanía de su muerte ${ }^{12}$. Y por último, a esta difícil evaluación se agregan las limitaciones de los padres a decidir por sus hijos al final de sus vidas, tema que ha sido analizado ante casos públicos ${ }^{13,14}$. Si para decidir sobre tratamientos se reconocen límites en la capacidad de los padres, con mayor razón se deberían considerar ante una petición de eutanasia. En el mismo sentido, la ley de derechos del paciente (Ley 20.584) establece que, en el caso de que el profesional tratante tenga dudas acerca de la competencia de la persona, deberá siempre solicitar la opinión del comité de ética del establecimiento.

A modo de conclusión, para responder si podría ser aceptable la eutanasia infantil, habría que decir que muy excepcionalmente $\mathrm{y}$, como se ha planteado en algunos países, sólo ante situaciones en las cuales hubiesen fracasado las medidas de cuidad o paliativo que se mencionaron anteriormente. $\mathrm{Y}$ ante igual pregunta para nuestro país, si bien el tema de la eutanasia está abierto a la deliberación ética ${ }^{15}$, frente a la eutanasia infantil la respuesta es que no podría ser aceptable, y que sólo podría ser discutida después de haber establecido políticas públicas, protocolos y servicios eficientes de cuidados paliativos para niños con enfermedades terminales, tanto oncológicas como no oncológicas.
Potenciales conflictos de interés: Este trabajo cumple con los requisitos sobre consentimiento/asentimiento informado, comité de ética, financiamiento, estudios animales y sobre la ausencia de conflictos de intereses según corresponda.

\section{Referencias}

1.- Service Public Federal Justice, 28 Fevr. 2014, Sénat de Belgique: Loi modifiant la loi du 28 mai 2002 relative à l'euthanasie, en vue d'étendre l'euthanasie aux mineurs. Disponible en: <http://www.ejustice.just.fgov. be/cgi/article_body.pl?language $=$ fr\&caller $=$ summary \&pub_date $=2014-03-12 \&$ numac $=2014009093>$

2.- Montero F: Repensar la eutanasia y el suicidio médicamente asistido. En Beca JP y Astete C. Bioética Clínica. Editorial Mediterráneo, Santiago, Chile 2012; pp 498510 .

3.- Grupo de Estudio, Declaración del Instituto Borja de Bioética (Universidad Ramon LLull): Hacia una posible despenalización de la eutanasia. Bioética-Debat.Org 2005. Disponible en http://www.bioetica-debat.org/ modules/news/article.php?storyid $=45$

4.- Leiva A: La Regulación de la Eutanasia, según la Ley № 20.584 sobre Derechos del Paciente. Rev Der PUCV. 2013; 41: 505-58. Disponible en: http://www.scielo.cl/ pdf/rdpucv/n41/a15.pdf

5.- Roxin $C$ : Tratamiento jurídico-penal de la eutanasia. Revista Electrónica de Ciencia Penal y Criminología. 1999; 01-10. Disponible en: http://criminet.ugr.es/recpc/ recpc_01-10.html

6.- Burlinger $N$, et al: Facing Persistent Challenges in Pediatric Decision-Making: New Hastings Center Guidelines. Pediatrics 2013; 132: 789-91.

7.- Clark JD, Dudzinski DM: The culture of dysthanasia: attempting CPR in terminally ill children. Pediatrics 2013; 131 (3): 572-80.

8.- Sans Sabrafen J, Abel Fabre F: Obstinación Terapéutica. Documento de la Real Academia de Medicina de Cataluña; 2005. Disponible en: http://www.samfyc.es/ pdf/GdTBio/201027.pdf

9.- Hernando P, Diestre G, Baigorr F: De la limitación de esfuerzo terapéutico a la adecuación del esfuerzo terapéutico. En Beca JP y Astete C. Bioética Clínica. Editorial Mediterráneo, Santiago, Chile 2012; pp $397-$ 405.

10.- Goic A: Apuntes sobre la eutanasia. Rev Med Chile 2005; 113: 371-5.

11.- Ossandón M: El derecho a rechazar tratamientos médicos ¿un reconocimiento del derecho a disponer de la propia vida? Revista de Derecho Público Iberoameri- 
BECA J. y cols.

cano. Ed. Universidad del Desarrollo, Santiago, Chile 2013; pp. 153-204.

12.- Gracia D, Jarobo Y, Espíldora N, et al: Toma de decisiones en el paciente menor de edad. Med Clin (Barc) 2001; 117: 179-1909

13.- Mc Nab ME, Beca JP: ¿Existen límites en las decisiones de los padres sobre el tratamiento de sus hijos? Rev Chil
Pediatr 2010; 81: 536-40.

14.- Couceiro A: Lectura clínica de una sentencia judicial: el derecho a la vida y su aplicación en la toma de decisiones clíicas. Rev Chil Pediatr 2013; 84: 205-17.

15.- Grupo de estudios de Ética Clínica de la Sociedad Médica de Santiago: Eutanasia y acto médico. Rev Med Chile 2011; 139: 642-54. 